\title{
Balanced and Energy Efficient Multi Core- Aware Virtual Machine Placement Algorithm Using Multi-Dimensional Resource Space Partition Model
}

\author{
Nagadevi ${ }^{\mathrm{a}, 1}$ and Kasmir Raja ${ }^{\mathrm{b}}$ \\ ${ }^{a}$ Assistant Professor, Dept of ECE, SRM Institute of Science and Technology, Chennai, \\ India \\ ${ }^{b}$ UG Scholar, Dept of ECE, SRM Institute of Science and Technology, Chennai, India
}

\begin{abstract}
Optimal resource management is required in a data center to allocate the resources to users in a balanced manner. Balanced resource allocation is one of the key challenges in the data center. The multi-dimensional resources of a data center must be allocated in a balanced manner in all the dimensions of physical machines. The unbalanced resource allocation leads to unused residual resource fragments. The unused residual resource fragments leads to resource wastage. If the multidimensional data center resources are allocated in a balanced manner, the resource wastage does not occur. Also, the balanced allocation improves the power consumption. The balanced resource allocation reduces the resource wastage as well as reduces the power consumption. In this paper, we have designed a Balanced Energy Efficient Multi-Core Aware Virtual Machine Placement algorithm (MCA-BEE-VMP) using multi-dimensional resource space partition model to balance the resources like CPU and memory and also to reduce the power consumption. We used Google Cloud Jobs (GoCJ) dataset for the simulation. In our simulation of MCA-BEE-VMP using Cloud Sim simulation tool we have achieved balanced CPU and memory resources allocation in two dimensions of a physical machine. The resource wastage and power consumption is improved and the simulation results were analyzed.
\end{abstract}

Keywords: Multi-core, Virtual Machine Placement, Data Center, Multidimensional, Space partition model, balanced, energy efficient, Google Cloud Jobs, Cloud Sim

\section{Introduction}

Nowadays, the end users of a cloud computing run their applications and store their data in the data center(DC). The Cloud Service Providers provision the resources (CPU, Memory, Network etc.) requested by the users in the form of a virtual machines (VM). e.g. Amazon EC2 instances.

\footnotetext{
${ }^{1}$ Nagadevi, Assistant Professor, Dept of ECE, SRM Institute of Science and Technology, Chennai, India.
} Email:nagadevs@srmist.edu.in ${ }^{1}$ 
The user requested virtual machines are created in suitable physical machines (PM) of a data center. The process of creating a VM on a suitable PM is popularly known as Virtual Machine Placement (VMP). The optimal placement of VM on PM leads to save resources, improve power consumption and resource utilization. Considering the PMs of a data center as a multi-dimensional resources in the dimensions like, CPU, Memory, Storage, the process of VMP must place VMs in such a way that, any VMP must balance the PMs in all the dimensions of it.

\section{Background}

The main objectives of the VMP problem is to reduce power consumption by reducing number of running physical machines, to do dynamic resource allocation, to improve resource utilization, to minimize a cost of a data center, to improve SLA, to reduce number of VM migrations, to reduce resource wastage. The VMP could be categorized as, Single Dimensional VM Placement-Only the computational demand and computational capacity is considered for VMP.VM is placed if CPU requirement is less than the total available CPU capacity of a PM Multi-Dimensional VM Placement-Not only computational capacity , also, the memory, storage ,bandwidth, network etc., are considered.VM is placed if resource requirements satisfies the resources in all dimensions.

\section{Related Work}

Earlier, many algorithms have been proposed by authors[1]-[5] for VMP, but the physical cores (pCPU) of a PM is not considered except[6], [7]. In most of the existing VMP algorithms authors have considered the total CPU capacity of a PM not the pCPU capacity of a PM for VMP .Resource usage and power calculation is calculated for virtual machine placement.[8].Energy efficiency is discussed in [5], [9],[3], [10] [13$15]$.

\section{Proposed System Model}

We have deigned our algorithm based on, placing ' $n$ ' number of virtual machines on ' $\mathrm{m}$ ' number of physical machines. Consider, there are, $\mathrm{m}$ number of physical machines, such as PM-list $=\left\{\mathrm{pm}_{1}, \mathrm{pm}_{2} \ldots \mathrm{pm}_{\mathrm{m}}\right\}$ and $\mathrm{n}$, number of virtual machines such as VMlist $=\left\{\mathrm{vm}_{1}, \mathrm{vm}_{2} \ldots \mathrm{vm}_{\mathrm{n}}\right\}$.

So, the overall CPU Utilization of $\mathrm{m}$, number of PMs is calculated as,

$$
\text { CPU Utilization }=\frac{\text { Total Number of MIPS used by all Active Cores of a PM }}{\text { Total CPU (MIPS) capacity of a PM }}
$$

Similarly, the overall Memory Utilization of m, number of PMs is calculated as,

$$
\text { Memory Utilization }=\frac{\text { Total amount of RAM used by all Active Cores of a PM }}{\text { Total RAM capacity of the PM }}
$$


The overall Power Consumption of a cloud data center is,

$$
\sum_{j=1}^{m} \text { Power }=\sum_{j=1}^{m} P^{j} \cdot\left[\left(\text { pow }_{j}^{\text {busy }}-\text { pow }_{j}^{i d l e}\right) \frac{\sum_{i=1}^{n} v m_{i}^{m i p s} \cdot x_{i j}}{\text { pm }_{j}^{n c o r e s} p m_{j}^{c-m i p s}}+\text { pow }_{j}^{\text {idle }}\right]
$$

where $\sum_{j=1}^{m}$ Power is the power consumption of $\mathrm{m}$, number of PMs in proposed system. $p o w_{j}^{\text {busy }}{ }^{\text {, }}$ pow $w_{j}^{\text {idle }}$ are the power consumption of $\mathrm{pm}_{\mathrm{j}}$ at busy and idle time. $P^{j}$, specifies that the physical machine $\mathrm{pm}_{\mathrm{j}}$ is active. The potential cost of resource wastage of a PM in a data center, in multiple dimensions like CPU, memory, disk and so on, is calculated as ,

$$
W_{j}=\frac{\left|L_{j}^{p}-L_{j}^{m}\right|+\epsilon}{U_{j}^{p}+U_{j}^{m}}
$$

where $U_{j}^{p}$ and $U_{j}^{m}$ are the normalized CPU and memory resource usage. $L_{j}^{p}$ and $L_{j}^{m}$ are the normalized remaining CPU and memory resource. $\varepsilon$, is the very small positive real number and its value is set to 0.0001 .

The requirement is to find a map

\section{Map F: VM-list $\rightarrow$ PM-list}

between PM-list and VM-list such that to satisfy all the below mentioned objective functions.

The objective function our proposed algorithm is,

$$
\min \sum_{j=1}^{m} R W
$$

$$
\min \sum_{j=1}^{m} \text { Power }
$$

where $\mathrm{RW}$, is the resource wastage of m number of physical machines and Power, is the power consumption of $\mathrm{m}$, number of physical machines

\section{Balanced Energy Efficient Multi-Core-Aware VMP algorithm using Multi- Dimensional resource space partition Model (MCA-BEE-VMP)}

We have proposed MCA-BEE-VMP, algorithm using the multi-dimensional resource space partition model [8], [11], [12] to balance the resources in $\mathrm{d}=2$, dimensions of a $\mathrm{PM}$.

\subsection{Multi-Dimensional Space Partition Model}

$\mathrm{PM}$ is the multi-dimensional resource .To represent the PM as a multi-dimensional resource, a vector space partition model shown in Fig.1 is used for our proposed MCABEE-VMP. Each PM is represented as a point, $\mathrm{P}\left(R U_{j}^{c p u}, R U_{j}^{m e m}\right)$, in a two dimensional $\mathrm{X}, \mathrm{Y}$ axis, by taking CPU capacity on $\mathrm{X}$-axis and memory capacity on $\mathrm{Y}$ axis. A new point NP $\left(R U R_{j}^{c p u}, R U R_{j}^{m e m}\right)$ in the resource space is determined for PM, in which the PM will settle after placing a new VM. Fig.1 shows the multi-dimensional space partition model in which different domains of resource space is formulated. The domains in the model are Acceptance Domain (AD), Balanced Domain (BD) and 
Unbalanced Domain (UD). The domain to which the PM belongs is determined by the position of the corresponding point $\mathrm{P}$ and NP.AD has the highest priority for being selected for VMP .In AD the resources nearly exhausted and PMs have small resource wastage in it.BD has a second highest priority for VMP and is a balanced space to place a VM.UD indicates the worst case resource wastage for a PM and it is avoided in our algorithm for VMP. The Resource Utilization (RU) of a PM in dimensions CPU and memory is calculated as a current state vector point, $\mathrm{P}\left(R U_{j}^{c p u}, R U_{j}^{m e m}\right)$ as,

$$
C_{j}^{c p u}=p m_{j}^{c-m i p s} \cdot p m_{j}^{n c o r e s} R U_{j}^{c p u}=\frac{x_{i j} . \sum_{i=1}^{n} v m_{i}^{\text {mips }}}{C_{j}^{c p u}} R U_{j}^{\text {mem }}=\frac{x_{i j} . \sum_{i=1}^{n} v m_{i}^{\text {mem }}}{p m_{j}^{\text {mem }}}
$$

where $C_{j}^{c p u}$ is the total CPU capacity of a $\mathrm{pm}_{\mathrm{j}}, R U_{j}^{c p u}$ is the CPU resource utilization of $\mathrm{pm}_{\mathrm{j}}$ and $R U_{j}^{\text {mem }}$ is the memory resource utilization of $\mathrm{pm}_{\mathrm{j}}$. Resource Usability Ratio (RUR) determines whether the PM is good enough to hold a new VM according to the space partition model. So, residual resource fragments of PM are reduced. A residual resource fragment which increases the resource wastage in a PM is minimized. Thus the RUR is a metric to determine the probable placement status of a PM.RUR is calculated for all PMs after placing a new VM, in resource dimensions CPU and memory as new state vector point, $\mathrm{NP}\left(R U R_{j}^{c p u}, R U R_{j}^{\text {mem }}\right)$ as,

$$
R U R_{j}^{c p u}=\frac{C_{j}^{c p u} \cdot R U_{j}^{c p u}+V M_{n e w}^{\text {mips }}}{C_{j}^{c p u}} \quad R U R_{j}^{\text {mem }}=\frac{p m_{j}^{\text {mem }} \cdot R U_{j}^{c p u}+V M_{\text {new }}^{\text {mem }}}{p m_{j}^{\text {mem }}}
$$

where $R U R_{j}^{c p u}$ is the CPU resource usability ratio of $\mathrm{pm}_{\mathrm{j}}, R U R_{j}^{m e m}$ is the memory resource usability ratio of $\mathrm{pm}_{\mathrm{j}}, V M_{\text {new }}^{\text {mips }}$ is the MIPS requirement of a new VM and $V M_{n e w}^{\text {mem }}$ is the memory demand of new VM.Given the point $\mathrm{P}\left(R U_{j}^{c p u}, R U_{j}^{\text {mem }}\right)$ and the parameters $\mathrm{r} 0$ and $\mathrm{R} 0$, the domain in which the PM lies is determined as a function $f(P)$ and is given as,

$$
f(P)=\left\{\begin{array}{rr}
A D, & \text { if } \operatorname{distance}(P, E) \leq r 0 \\
B D, & \text { if } R U_{j}^{\text {cpu }} \text { and } R U_{j}^{\text {mem }} \leq(1-R 0) \\
U D, & \text { otherwise }
\end{array}\right.
$$

where, $\operatorname{distance}(P, E)$ is the distance between the two points $\mathrm{P}$ and $\mathrm{E}(1,1), \mathrm{r} 0$ is the satisfaction factor usually set to 0.0 , just to ensure the idea of balancing in multidimensional resources and $\mathrm{R} 0$ is the balance factor. The PM selection is very much affected by R0.

\subsection{MCA-BEE-VMP Algorithm}

MCA-BEE-VMP algorithm is shown in Algorithm 1.Initially, the PMs with the available pCPU capacity and memory is selected for placing a VM. For each PM results from step 1, the PM is selected for step 3, if anyone of the pCPU is sufficient enough to hold the new VM along with the current CPU load. For the PMs results from step 2, the point $\mathrm{P}$ and $\mathrm{NP}$ is determined .If $\mathrm{P}$ is in $\mathrm{AD}$ or NP is in $\mathrm{BD}$, then the first PM is selected as a target PM and first pCPU of that PM that has enough resource to place $\mathrm{VM}$, is selected as a target pCPU 
Algorithm1: Multi Core-Aware Balanced Energy Efficient Virtual Machine Placement Algorithm (MCA-BEE-VMP):

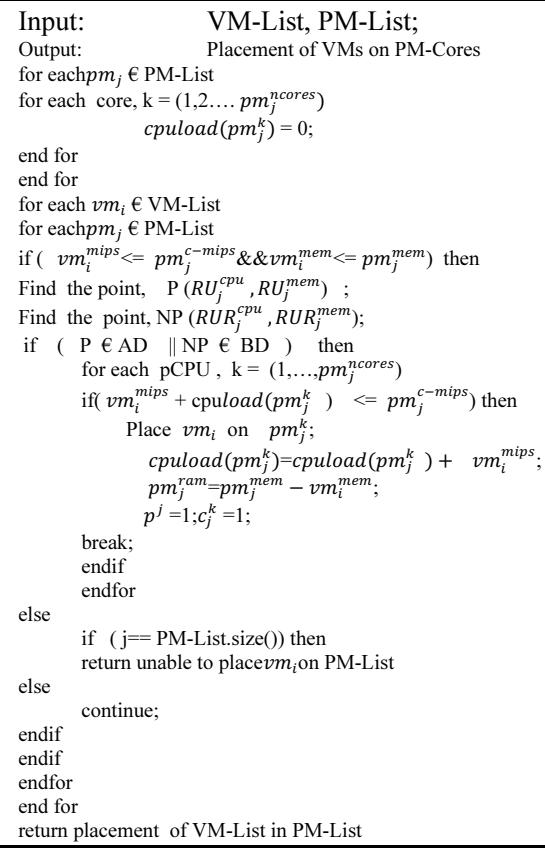

Algorithm 2: Multi Core-Aware Virtual Machine Placement Algorithm (MCA-VMP): We have compared our algorithm with MCA-VMP, where the virtual machines are placed on a PM based on First Fit strategy.

Algorithm 3: Multi Core-Aware Balanced Virtual Machine Placement Algorithm (MCAB-VMP):

We also have compared our proposed algorithm with MCAB-VMP, in which the distance between the VM and PM is calculated using Dot Product.

\section{Performance Evaluation}

The simulation results using MCA-BEE-VMP is shown in Figure .1.
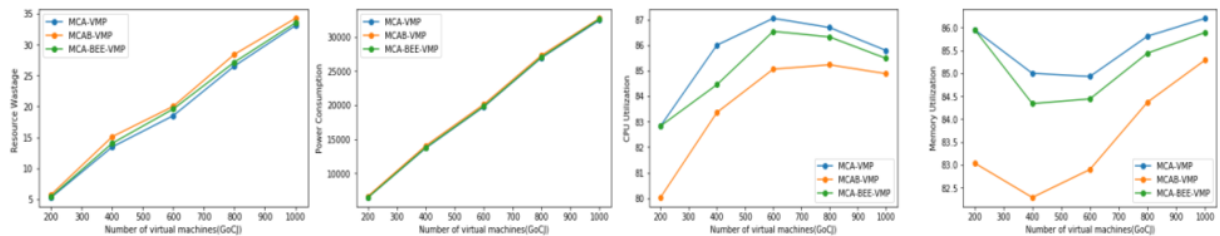

(a)Resource Wastage(b)Power Consumption in watts(c) CPU Utilization(d)Memory Utilization

Figure 1. Simulation results using MCA-BEE-VMP algorithm (a)Resource Wastage (b)Power Consumption in watts(c) CPU Utilization (d)Memory Utilization 


\section{Conclusion}

In this research, we have argued that core awareness is necessary in the process of VMP in cloud data centers. The non-core aware VM placement algorithms leads to sub-optimal results and PM overload. We designed a new algorithm based on cores called MCA-BEE-VMP algorithm using multi-dimensional resource partition model. Our simulation results shows that MCA-BEE-VMP is efficient in terms of resource wastage and power consumption. Also, the CPU and memory utilization of all the physical machines is balanced.

\section{References}

[1] Beloglazov.A and Buyya.R .Managing Overloaded Hosts for Dynamic Consolidation of Virtual Machines in Cloud Data Centers under Quality of Service Constraints. IEEE Trans. Parallel Distrib. Syst., vol. 24, no. 7, pp. 1366-1379, Jul. 2013, doi: 10.1109/TPDS.2012.240.

[2] Beloglazov.A and Buyya.R. Energy Efficient Allocation of Virtual Machines in Cloud Data Centers, in 2010 10th IEEE/ACM International Conference on Cluster, Cloud and Grid Computing, Melbourne, Australia, 2010, pp. 577-578, doi: 10.1109/CCGRID.2010.45.

[3] Abohamama.A.S and Hamouda.E.A hybrid energy-Aware virtual machine placement algorithm for cloud environments, Expert Systems with Applications, vol. 150, p. 113306, Jul. 2020, doi: 10.1016/j.eswa.2020.113306.

[4] Alouane.M and H. El Bakkali.Virtualization in Cloud Computing: Existing solutions and new approach, in 2016 2nd International Conference on Cloud Computing Technologies and Applications (CloudTech), Marrakech, Morocco, May 2016, pp. 116-123, doi: 10.1109/CloudTech.2016.7847687.

[5] Azizi.S, Shojafar.M,Abawajy.J, and R. Buyya, GRVMP: A Greedy Randomized Algorithm for Virtual Machine Placement in Cloud Data Centers, IEEE Systems Journal, pp. 1-12, 2020, doi: 10.1109/JSYST.2020.3002721.

[6] Z. Á. Mann, Allocation of Virtual Machines in Cloud Data Centers-A Survey of Problem Models and Optimization Algorithms, ACM Comput. Surv., vol. 48, no. 1, pp. 1-34, Sep. 2015, doi: $10.1145 / 2797211$.

[7] Mann.Z.A .Multicore-Aware Virtual Machine Placement in Cloud Data Centers. IEEE Trans. Comput., vol. 65, no. 11, pp. 3357-3369, Nov. 2016, doi: 10.1109/TC.2016.2529629.

[8] Gupta.M.K, A. Jain, and T. Amgoth, Power and resource-aware virtual machine placement for IaaS cloud, Sustainable Computing: Informatics and Systems, vol. 19, pp. 52-60, Sep. 2018, doi: 10.1016/j.suscom.2018.07.001.

[9] Beloglazov.A, Abawajy.J, and Buyya.R. Energy-aware resource allocation heuristics for efficient management of data centers for Cloud computing. Future Generation Computer Systems, vol. 28, no. 5, pp. 755-768, May 2012, doi: 10.1016/j.future.2011.04.017.

[10] Aruna.P and Vasantha.S . A Meta-Heuristic based Virtual Machine Placement and Migration for Optimal Resource Utilization in the Cloud Computing Environment, Asia. Jour. Rese. Soci. Scie. and Human., vol. 6, no. 5, p. 725, 2016, doi: 10.5958/2249-7315.2016.00148.9.

[11] X. Li, Z. Qian, S. Lu, and J. Wu, Energy efficient virtual machine placement algorithm with balanced and improved resource utilization in a data center, Mathematical and Computer Modelling, vol. 58, no. 5-6, pp. 1222-1235, Sep. 2013, doi: 10.1016/j.mcm.2013.02.003.

[12] W. Huang, X. Li, and Z. Qian, An Energy Efficient Virtual Machine Placement Algorithm with Balanced Resource Utilization, in 2013 Seventh International Conference on Innovative Mobile and Internet Services in Ubiquitous Computing, Taichung, Taiwan, Jul. 2013, pp. 313-319, doi: 10.1109/IMIS.2013.59.

[13] Ambeth Kumar.V.D (2017).Efficient Routing for Low Rate Wireless Network a Novel Approach. International Journal of Image Mining, Vol. 2, Nos. 3/4, 2017, 2017

[14] Nanagasabapathy .Ket.al. Validation system using smartphone luminescence. IEEE International Conference on Intelligent Computing, Instrumentation and Control Technologies (ICICICT), Pages: 235 - 239, 6-7 July 2017, Kannur, India

[15] Ambeth Kumar.V.D, Ashok Kumar.V.D, Divakar.H ,Gokul.R. Cloud enabled media streaming using Amazon Web Services.IEEE (ICSTM), Pages: 195 - 198, 2-4 Aug. 2017, India (DOI: $10.1109 /$ icstm.2017.8089150) 